\title{
BMJ Open Associations between objectively assessed and self-reported sedentary time with mental health in adults: an analysis of data from the Health Survey for England
}

\author{
Mark Hamer, ${ }^{1}$ Ngaire Coombs, ${ }^{1}$ Emmanuel Stamatakis ${ }^{1,2}$
}

To cite: Hamer M, Coombs N, Stamatakis E. Associations between objectively assessed and selfreported sedentary time with mental health in adults: an analysis of data from the Health Survey for England. BMJ Open 2014;4:e004580. doi:10.1136/bmjopen-2013004580

- Prepublication history and additional material is available. To view please visit the journal (http://dx.doi.org/ 10.1136/bmjopen-2013004580).

Received 29 November 2013 Revised 20 February 2014 Accepted 27 February 2014

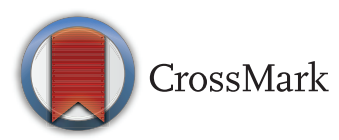

${ }^{1}$ Physical Activity Research Group, Department of Epidemiology and Public Health, University College London, London, UK

${ }^{2}$ Prevention Research Collaboration, School of Public Health, University of Sydney, Sydney, Australia

Correspondence to Dr Mark Hamer; m.hamer@ucl.ac.uk

\section{ABSTRACT}

Objective: There is increasing interest in the association between sedentary behaviour and mental health, although most studies have relied solely on self-reported measures, thus making results prone to various biases. The aim was to compare associations between objectively assessed and self-reported sedentary time with mental health in adults.

Setting: Community dwelling population sample drawn from the 2008 Health Survey for England.

Participants: 11658 (self-report analysis) and 1947

(objective data) men and women.

Primary outcome: The 12-item General Health Questionnaire was administered to assess psychological distress. Sedentary and physical activity (exposure) was objectively measured using accelerometers (Actigraph GT1M) worn around the waist during waking hours for seven consecutive days.

Results: The highest tertile of objective sedentary time was associated with higher risk of psychological distress (multivariate adjusted $\mathrm{OR}=1.74,95 \% \mathrm{Cl} 1.07$ to 2.83), as was the highest tertile of self-reported total sitting time ( $\mathrm{OR}=1.34,95 \% \mathrm{Cl} 1.15$ to 1.56$)$. Selfreported, but not objective, moderate-to-vigorous physical activity was associated with lower risk of psychological distress. Only objective light-intensity activity was associated with lower risk of psychological distress.

Conclusions: Sedentary time is associated with adverse mental health.

\section{INTRODUCTION}

Adults spend approximately $60-70 \%$ of their waking hours in sedentary activities, ${ }^{12}$ which are characterised by energy expenditure below 1.5 metabolic equivalents while in a sitting or reclined posture. There is increasing interest in the association between sedentary behaviour and mental health. ${ }^{3-12}$ Several longitudinal studies have demonstrated an

\section{Strengths and limitations of this study}

- Use of objective physical activity assessment.

- Large representative sample of the general population.

- The main limitation is the cross-sectional design.

- Future studies are required to examine the biological plausibility.

association of self-reported TV/computer time $^{7}$ and TV time alone ${ }^{8}$ with higher risk of mental disorders, including depression and anxiety, at follow-up. However, data from other studies suggest that not all types of sedentary behaviours are related to adverse mental health. ${ }^{59}$ For example, in a sample of older adults from the English Longitudinal Study of Ageing, TV time but not computer use was associated with higher depressive symptoms. ${ }^{9}$ Thus, it is unclear if the effects are being driven by physiological processes linked to excessive sitting or the contrasting environmental and social contexts in which they occur.

The majority of studies until now in this area have relied on self-reported measures of sedentary behaviours or total sitting time, thus making it difficult to tease apart associations between sedentary and mental health outcomes. To the best of our knowledge, only one population study has previously examined associations between objectively assessed sedentary time and depressive symptoms, which demonstrated null associations. ${ }^{13}$ Self-report is a potential limitation in this context as subjective mental state is a complex measure comprising cognitive and somatic symptoms, and thus self-reported mental health and sedentary behaviour might have conceptual overlap. Therefore, the aim of this study was to examine the association 
between objectively assessed and self-reported sedentary time with mental health in a population sample of adults. We hypothesised that if the effects are being driven by physiological processes linked to excessive sitting, we would observe consistent associations between objective and self-reported assessments of sedentary time with mental health. In contrast, if the associations were only observed for self-reported sedentary time, this might reflect a context-specific effect or reporting bias.

\section{METHODS}

\section{Sample and study design}

The Health Survey for England (HSE) is a continuous survey that annually draws a nationally representative general population sample of adults living in households. The sample is drawn using multistage stratified probability sampling with postcode sectors as the primary sampling unit and the Postcode Address File as the sampling frame for households. Stratification was based on geographical areas and not on individual characteristics of the population. In the present analysis, we used data from the 2008 HSE, which had a special focus on physical activity and fitness. ${ }^{14}$ In the 2008 HSE, the household response rate for the core sample was $64 \%$. These analyses considered participants aged between 16 and 95 years and over with valid data on all demographic, behavioural and clinical variables of interest.

\section{Assessment of sedentary time and physical activity}

Objective measures: A subsample of HSE 2008 participants were asked to wear a uniaxial accelerometer that records movement on the vertical axis, the Actigraph GT1M (Actigraph, Pensacola, Florida, USA), during waking hours for seven consecutive days. The accelerometer provides a measure of the frequency, intensity and duration of physical activity and allows classification of activity levels as sedentary, light, moderate-to-vigorous physical activity (MVPA). The raw accelerometry data were processed using specialist software (KineSoft, New Brunswick, Canada) to produce a series of standardised outcome variables. ${ }^{12}$ Only participants who wore the accelerometer for a minimum of $10 \mathrm{~h} /$ day were included in the present analyses. Although participants with at least 1 day of valid wear have been included in these analyses, the majority $(70 \%)$ had between 6 and 7 days and $84 \%$ had at least three valid days. We used the following cut-off points to calculate daily times in each activity intensity band: sedentary $(<1.5$ MET): 0-199 counts/min (CPM); light (1.5-3 MET) 2002019 CPM; MVPA (>3 MET): $\geq 2020$ CPM. $^{12}$ All physical activity and sedentary behaviour variables were converted to time (in minutes) per valid day.

Self-report: The self-reported measures have been described in detail elsewhere. ${ }^{1}{ }^{15}$ Briefly, sedentary behaviour was assessed using a set of questions enquiring about weekday and weekend time spent on (1) TV (including DVDs and videos) viewing and (2) any other sitting during non-work times, including reading and computer use. For those participants who were professionally active (ie, those who answered 'yes' to the question 'In the last 4 weeks, did you do any paid or unpaid work either as an employee or as selfemployed (including voluntary or part time work)?'), another set of questions assessed the average daily times spent sitting or standing while at work ('On an average workday in the last 4 weeks, how much time did you usually spend sitting down or standing up?'). Physical activity was assessed using the long version of the HSE questionnaire that was used in the 1997 survey for the first time and was repeated in the 1998, 2006 and 2008 surveys. Questions included frequency (number of days in the last 4 weeks) and duration (min/day) of participation in walking for any purpose and any recreational exercise (eg, cycling, swimming, aerobics, gym exercises, dancing, team sports or racket sports). Weekly self-reported MVPA hours/week was calculated as number of days of participation multiplied by time per day in each activity type. As with objectively assessed physical activity, tertiles of TV, non-TV leisuretime sitting, total sitting and MVPA time were derived for the analyses.

\section{Psychological distress}

Mental health was assessed using the 12-item version of the General Health Questionnaire (GHQ-12), a widely utilised measure of psychological distress in populationbased studies. ${ }^{16}{ }^{17}$ We employed a GHQ-12 cut-off score of $\geq 4$ to denote psychological distress. This definition has not only been validated against standardised psychiatric interviews, but has also been strongly associated with depression and anxiety. ${ }^{18}$

\section{Demographic and clinic variables}

Computer-assisted personal interviewing modules assessed respondents' demographics, occupational status, longstanding illness, alcohol consumption and smoking habits. Height and weight were also measured for the calculation of body mass index (BMI), computed as weight (kilograms) divided by squared height (metres).

\section{Statistical analyses}

Participants were categorised into tertiles for sedentary and activity categories. We used multiple logistic regression to compute ORs with accompanying $95 \%$ CI for the association between sedentary time/activity and psychological distress. The models were adjusted for potential confounding factors, including age, sex, smoking (never; previous; current), frequency of alcohol intake (at least 1/week; monthly; rarely or never), BMI (normal weight, $\mathrm{BMI}<25$; overweight, BMI 25-30; obese, BMI $\left.>30 \mathrm{~kg} / \mathrm{m}^{2}\right)$, social occupational group (professional and managerial occupations; skilled non-manual; routine and manual), highest educational qualification, (non-mental health-related) long-standing illness and Actigraph wear time (for analyses involving accelerometry). Finally, the models were mutually adjusted for MVPA in the analyses using sedentary or 
light activity as the main exposure, or sedentary when using MVPA as the main exposure. Models were run for each main exposure including an interaction term between the main exposure and sex. This interaction term was not significant in any of the models, so analysis was sex adjusted, but not sex stratified. The complex samples module in SPSS was used to take into account the survey design, which adjusted for the uneven non-response and accounted for the clusters and stratum used in data collection. A sensitivity analysis was run for accelerometrymeasured sedentary time, using a more conservative cut-off of $<100$ CPM. Owing to the large difference in sample size between accelerometry and self-reported outcomes, a second sensitivity analysis was conducted to test whether observed differences in results between accelerometry and self-reported exposures were due to differences between the accelerometry and non-accelerometry samples. Logistic regression models were run substituting sedentary/physical activity tertiles for sample type (accelerometry/non-accelerometry sample). All analyses were conducted using SPSS V.21.

\section{RESULTS}

\section{Descriptives}

Of the self-report sample used in the analyses (11 658), $12.7 \%$ reported psychological distress (see table 1 ). Respondents with psychological distress were more likely to be female, from a lower social class, have a lower educational qualification, be out of paid work, smoke, report a non-mental health long-standing illness, and have problems with usual activities than those without psychological distress (all $\mathrm{p}<0.001$ ). Respondents with psychological distress were also more sedentary and spent on average 31 and $25 \mathrm{~min} /$ day more in TV and non-TV leisure-time sitting, respectively, and $10 \mathrm{~min} /$ day less in self-reported MVPA $(\mathrm{p}<0.001)$.

\section{Accelerometry-measured sedentary time and physical activity}

Table 2 presents multivariable-adjusted associations between accelerometry-measured sedentary time and physical activity, with psychological distress. Sedentary time ( $<200$ CPM) was directly associated with psychological distress after adjustment for all covariables including MVPA, although this was more apparent in the highest tertile (OR=1.74, 95\% CI 1.07 to 2.83). Light activity (200-2018 CPM) was inversely associated with risk for psychological distress, although the association was not linear. MVPA, however, was not associated with psychological distress in any models.

\section{Self-reported sedentary time and physical activity}

Table 3 presents the associations between self-reported sedentary time and physical activity with psychological distress. Total sitting time was directly associated with risk of psychological distress, although only the highest tertile of sitting was different to the referent group $(\mathrm{OR}=1.34,95 \% \mathrm{CI} 1.15$ to 1.56$)$. This association was

Table 1 Sample characteristics in relation to psychological distress

\begin{tabular}{|c|c|c|c|c|c|c|c|}
\hline \multirow[b]{2}{*}{ Categorical variables } & & \multicolumn{2}{|l|}{$\mathrm{GHQ}<4$} & & \multicolumn{2}{|l|}{$\mathrm{GHQ} \geq 4$} & \multirow[b]{2}{*}{ p Value* } \\
\hline & & Per cent & $\mathbf{N}$ & & Per cent & $\mathbf{N}$ & \\
\hline Sex (\% male) & & 48.1 & 10172 & & 38.2 & 1486 & $<0.001$ \\
\hline Social class (\% semi-skilled manual or lower) & & 21.7 & 10172 & & 26.4 & 1486 & $<0.001$ \\
\hline Qualification (\% secondary school or lower) & & 50.8 & 10172 & & 58.1 & 1486 & $<0.001$ \\
\hline Percentage not in paid work & & 33.9 & 10172 & & 53.6 & 1486 & $<0.001$ \\
\hline Smoking (\% current) & & 20.6 & 10172 & & 29.9 & 1486 & $<0.001$ \\
\hline Alcohol consumption ( $\%$ 5+ times a week) & & 18.5 & 10172 & & 16.4 & 1486 & $<0.001$ \\
\hline Percentage with LSI† (non-mental health) & & 39.8 & 10172 & & 57.3 & 1486 & $<0.001$ \\
\hline Percentage of problems with usual activities & & 10.4 & 10172 & & 40.5 & 1486 & $<0.001$ \\
\hline Continuous variables & $\mathbf{M}$ & SD & $\mathbf{N}$ & $\mathbf{M}$ & SD & $\mathbf{N}$ & $\mathbf{p}$ \\
\hline Age (years) & 50.0 & $(17.6)$ & 10172 & 48.3 & $(17.2)$ & 1486 & 0.149 \\
\hline BMI $\left(\mathrm{kg} / \mathrm{m}^{2}\right)$ & 27.3 & $(5.0)$ & 10172 & 27.8 & $(6.0)$ & 1486 & 0.046 \\
\hline TV viewing (min/day) & 167.9 & (99.6) & 10172 & 199.3 & $(133.1)$ & 1486 & $<0.001$ \\
\hline Non-TV leisure-time sitting (min/day) & 126.7 & (92.0) & 10172 & 152.0 & $(119.0)$ & 1486 & $<0.001$ \\
\hline Total leisure-time sitting (min/day) & 294.6 & $(136.8)$ & 10172 & 351.3 & $(17.4)$ & 1486 & $<0.001$ \\
\hline Self-reported MVPA (min/day) & 45.7 & $(67.2)$ & 10172 & 35.3 & (63.7) & 1486 & $<0.001$ \\
\hline Accelerometry sedentary time (min/day) & 578.5 & (93.2) & 1698 & 574.1 & (98.1) & 249 & 0.386 \\
\hline Accelerometry light physical activity time (min/day) & 293.9 & (85.3) & 1698 & 288.6 & (90.5) & 249 & 0.436 \\
\hline Accelerometry MVPA time (min/day) & 29.2 & (25.5) & 1698 & 25.7 & (22.4) & 249 & 0.027 \\
\hline Accelerometry wear time per valid day (min) & 835.9 & (74.7) & 1698 & 822.8 & (77.3) & 249 & 0.004 \\
\hline
\end{tabular}


Table 2 Multivariable-adjusted associations between accelerometry-measured sedentary time, light intensity activity and MVPA, with psychological distress

\begin{tabular}{|c|c|c|c|c|}
\hline & $\mathbf{N}$ & Model $1^{*}$ & Model 2† & Model 3‡ \\
\hline \multicolumn{5}{|c|}{ Tertiles of sedentary time } \\
\hline Low & 649 & 1.00 & 1.00 & 1.00 \\
\hline Med & 649 & $1.00(0.66$ to 1.53$)$ & $1.05(0.68$ to 1.62$)$ & $1.09(0.70$ to 1.71$)$ \\
\hline High & 649 & 1.52 (0.98 to 2.35$)$ & 1.59 (1.01 to 2.51$)$ & 1.74 (1.07 to 2.83$)$ \\
\hline p Value & & 0.072 & 0.071 & 0.037 \\
\hline \multicolumn{5}{|c|}{ Tertiles of light PA time } \\
\hline Low & 649 & 1.000 & 1.000 & 1.000 \\
\hline Med & 649 & $0.62(0.42$ to 0.92$)$ & $0.56(0.37$ to 0.84$)$ & $0.56(0.37$ to 0.84$)$ \\
\hline High & 649 & $0.79(0.53$ to 1.17$)$ & $0.74(0.49$ to 1.12$)$ & $0.73(0.48$ to 1.12$)$ \\
\hline p Value & & 0.056 & 0.021 & 0.020 \\
\hline \multicolumn{5}{|c|}{ Tertiles of MVPA } \\
\hline Low & 649 & 1.000 & 1.000 & 1.000 \\
\hline Med & 649 & $0.67(0.45$ to 0.99$)$ & $0.81(0.54$ to 1.22$)$ & 0.90 (0.59 to 1.37$)$ \\
\hline High & 649 & $0.84(0.55$ to 1.28$)$ & 1.05 (0.68 to 1.62$)$ & 1.27 (0.80 to 2.04$)$ \\
\hline p Value & & 0.130 & 0.432 & 0.283 \\
\hline
\end{tabular}

${ }^{*}$ Model 1 adjusted for age, sex and accelerometry wear time.

†Model 2 also adjusted for smoking, alcohol, education, BMI and social occupational group employment long-standing illness (non-mental only).

$\ddagger$ Model 3 also adjusted for tertiles of accelerometry-measured MVPA (for sedentary and light PA exposures) or sedentary time (for MVPA exposure).

BMI, body mass index; MVPA, moderate-to-vigorous physical activity; PA, physical activity.

largely driven by 'non-TV viewing' sedentary time as the associations for TV viewing demonstrated an inconsistent pattern. MVPA was inversely associated with risk of psychological distress in a dose-response manner $(p<0.001$ for all models).

\section{Sensitivity analysis}

In the sensitivity analysis using a different sedentary cut-off of 100 CPM (see online supplementary table S1), similar results were found (multivariate adjusted OR of psychological distress for the highest tertile of sedentary

Table 3 Multivariable-adjusted associations between self-reported total sitting time, sedentary behaviours and MVPA with psychological distress

\begin{tabular}{|c|c|c|c|c|}
\hline & $\mathbf{N}$ & Model $1^{*}$ & Model 2† & Model 3ł \\
\hline \multicolumn{5}{|c|}{ Tertiles of total sitting } \\
\hline Low & 3836 & 1.00 & 1.00 & 1.00 \\
\hline Med & 3910 & $1.11(0.95$ to 1.29$)$ & $0.99(0.85$ to 1.16$)$ & $0.97(0.83$ to 1.13$)$ \\
\hline High & 3912 & 2.07 (1.79 to 2.38$)$ & $1.41(1.21$ to 1.64$)$ & $1.34(1.15$ to 1.56$)$ \\
\hline$p$ Value & & $<0.001$ & $<0.001$ & $<0.001$ \\
\hline \multicolumn{5}{|c|}{ Tertiles of TV time } \\
\hline Low & 3304 & 1.00 & 1.00 & 1.00 \\
\hline Med & 4432 & 0.89 (0.77 to 1.03$)$ & $0.84(0.72$ to 0.98$)$ & $0.83(0.71$ to 0.97$)$ \\
\hline High & 3922 & $1.56(1.35$ to 1.80$)$ & $1.14(1.00$ to 1.35$)$ & $1.11(0.95$ to 1.30$)$ \\
\hline $\mathrm{p}$ Value & & $<0.001$ & $<0.001$ & $<0.001$ \\
\hline \multicolumn{5}{|c|}{ Tertiles of non-TV leisure-time sitting } \\
\hline Low & 4208 & 1.00 & 1.00 & 1.00 \\
\hline Med & 3673 & 0.99 (0.86 to 1.15$)$ & $0.95(0.82$ to 1.10$)$ & $0.95(0.82$ to 1.10$)$ \\
\hline High & 3777 & 1.55 (1.35 to 1.77$)$ & 1.26 (1.09 to 1.45$)$ & 1.23 (1.07 to 1.42$)$ \\
\hline$p$ Value & & $<0.001$ & $<0.001$ & 0.001 \\
\hline \multicolumn{5}{|c|}{ Tertiles of weekly MVPA } \\
\hline Low & 3876 & 1.000 & 1.000 & 1.000 \\
\hline Med & 3864 & 0.56 (0.49 to 0.65$)$ & 0.69 (0.59 to 0.79$)$ & $0.70(0.60$ to 0.81$)$ \\
\hline High & 3918 & $0.52(0.45$ to 0.60$)$ & 0.63 (0.54 to 0.73$)$ & 0.65 (0.56 to 0.76$)$ \\
\hline $\mathrm{p}$ Value & & $<0.001$ & $<0.001$ & $<0.001$ \\
\hline
\end{tabular}

${ }^{*}$ Model 1 adjusted for age, sex and accelerometry wear time.

†Model 2 also adjusted for smoking, alcohol, education, BMI and social occupational group employment long-standing illness (non-mental only).

¥Model 3 also adjusted for tertiles of self-reported MVPA (for TV and sitting exposures) or total sitting time (for MVPA exposure). BMI, body mass index; MVPA, moderate-to-vigorous physical activity. 
time compared to the lowest tertile $=2.04$, 95\% CI 1.29 to $3.21, \mathrm{p}=0.005)$. We also examined if there were differences in prevalence of psychological distress between the accelerometry sample and main sample (see online supplementary table S2). Sample type (accelerometry vs non-accelerometry), however, was not a significant predictor of psychological distress, after adjusting for relevant covariates (age, sex, smoking, employment status, long-standing illness and self-reported MVPA and TV time). Lastly, we examined the influence of the number of valid Actigraph wear days, but there were no associations found with psychological distress as the outcome.

\section{DISCUSSION}

The aim of this study was to examine the association between objectively assessed and self-reported sedentary time with mental health in a population sample of adults. Our findings consistently show an association between sedentary time and adverse mental health whether using objective or self-reported measures of sedentary time. Nevertheless, in contrast to previous evidence, ${ }^{4} 589$ the associations between context specific sedentary time (TV viewing) and psychological distress were far less consistent. Given that subjective mental state is a complex measure comprising cognitive (depressed mood) and somatic symptoms (eg, lethargy, tiredness, lack of appetite and pain), this might partly influence an individual's assessment of context specific sedentary time. Our findings are not consistent with data from the National Health and Nutrition Examination Survey (NHANES) that showed null associations between objectively assessed sedentary time and depressive symptoms in the main sample, although they did find an association among overweight/ obese adults in sensitivity analyses. ${ }^{13}$ Nevertheless, our study used a composite measure of psychological distress consisting of items on anxiety and depression; thus, our data cannot be directly compared to the measure of depressive symptoms used in NHANES.

There is mounting evidence to suggest detrimental effects of excess sedentary time on mental health, although plausible biological mechanisms are currently lacking. There are numerous data showing associations between sedentary time and cardiometabolic risk factors $^{2}{ }^{19}{ }^{20}$; thus, the links with mental health might act partly through these mechanisms. In particular, the role of low-grade inflammation in depression has gained substantial attention, ${ }^{21}$ although in a recent study $\mathrm{C}$ reactive protein did not explain the link between TV viewing and depressive symptoms in older adults. ${ }^{22}$ Psychosocial mechanisms might also be important. For example, passive sedentary activities such as TV viewing might encourage social isolation and limit the development of social networks.

We are not aware of any other studies that have compared associations of objective and self-reported sedentary/MVPA in relation to mental health outcomes. There was a consistent association between sedentary time and adverse mental health whether using objective or self-reported measures, albeit stronger with the objective measure. In contrast, we observed a discrepancy in results between self-reported and objectively assessed MVPA in relation to psychological distress, showing associations only for self-reported measures. In our recent studies, where we also compared accelerometry and self-reported exposures but in relation to cardiometabolic outcomes, ${ }^{1}{ }^{14}$ we found associations between MVPA and most outcomes for self-reported and objective measures. Thus, one interpretation of the present results is that self-reported mental health and MVPA might have conceptual overlap causing participants with poor mental health to misreport their activity levels. For example, symptoms such as lethargy may cause individuals to under-report their activity. In addition, cognitive impairment that is sometimes associated with depression $^{23}$ could impair recall, introducing bias into the results. Nevertheless, one might view objective and selfreported activity as different measures since objective assessment cannot take context into account and, by definition, measure slightly different aspects of MVPA. In this regard, context might be extremely important as some of the effects of physical activity on mental health are most likely driven by factors such as social interaction, whereas accelerometry is simply a measure of body movement and cannot capture contextual information such as 'where' and 'who with'. We did not, however, take contextual information into account in our analysis of self-reported MVPA.

Few studies have examined associations between objectively assessed physical activity and mental health, and those that have reveal inconsistent findings. For example, in a small cohort of elderly Japanese participants, physical activity was assessed objectively over 1 year, and inverse associations of activity with depression and stressful life events were observed. ${ }^{24}{ }^{25}$ In NHANES, an inverse dose response association was observed between MVPA and depressive symptoms. ${ }^{13}$ In a sample of 40 healthy women who completed a once-a-day mood rating scale for 1 week, we found inverse associations of depressive symptoms with objectively assessed light and moderate intensity activity but not vigorous. ${ }^{26}$ Other evidence is also equivocal. ${ }^{27} 28$ The present findings suggested that only objectively assessed light intensity activity was associated with lower risk of psychological distress, which is consistent with prior evidence showing associations between objective light-intensity physical activity and self-rated health in older adults. ${ }^{29}$ Data from randomised controlled trials also show that light/moderate intensity exercise has greater antidepressive effects, ${ }^{30}$ effects on positive $\operatorname{mood}^{31}$ and on reducing symptoms of fatigue compared with vigorous intensity. ${ }^{32} 33$ Lighter intensity activity may be more beneficial for mental health as greater exertion during vigorous forms of exercise may produce discomfort and shortness of breath. Our findings therefore suggest that modifying the balance between sedentary time and light intensity 
activity could be beneficial for mental health, as suggested by other recent studies. ${ }^{10} 29$

The main limitation of this study is the cross-sectional design, which precludes us from making any inferences about direction or causality. Sedentary behaviour has been longitudinally associated with risk of future depression in some, ${ }^{78}$ but not all, ${ }^{9}{ }^{11}$ studies; thus, the issue of causality remains unclear. Second, since accelerometry measures were only collected over 1 week, we do not know if this reflects habitual sedentary patterns in contrast to self-reported questions that enquired about activity over the past 4 weeks. However, strong test-retest reliability for MVPA ( $\mathrm{r}=0.89$ for men and $\mathrm{r}=0.76$ in women) was demonstrated in our validation study of 106 British adults from the general population, who wore accelerometers for two non-consecutive weeks over a month period. ${ }^{34}$ Undoubtedly, controlled trials are the best test of causality. However, studies of community samples have several advantages in that they are more representative. In the present study, we aimed to minimise possible confounding by controlling for key covariables. Future studies are required to examine the biological plausibility of a possible association between sedentary behaviour and mental health, which would further our understanding of this area.

Taken together, observational studies of representative community samples are an important approach for establishing links between sedentary behaviour and health outcomes, although further work is required to establish if the existing evidence reflects causal associations.

Contributors $\mathrm{MH}$ had full access to the data, and takes responsibility for the integrity and accuracy of the results. NC performed all statistical analyses under supervision of ES. MH drafted the manuscript. All authors contributed to the concept and design of the study and critical revision of the manuscript.

Funding This research received no specific grant from any funding agency in the public, commercial or not-for-profit sectors.

Competing interests $\mathrm{MH}$ is supported by the British Heart Foundation (RE/ 10/005/28296). ES is supported by a National Institute for Health Research Career Development award.

Patient consent Obtained.

Ethics approval Ethical approval for the 2008 survey was obtained from the Oxford A Research Ethics Committee (reference number 07/H0604/102).

Provenance and peer review Not commissioned; externally peer reviewed.

Data sharing statement No additional data are available.

Open Access This is an Open Access article distributed in accordance with the Creative Commons Attribution Non Commercial (CC BY-NC 3.0) license, which permits others to distribute, remix, adapt, build upon this work noncommercially, and license their derivative works on different terms, provided the original work is properly cited and the use is non-commercial. See: http:// creativecommons.org/licenses/by-nc/3.0/

\section{REFERENCES}

1. Stamatakis $\mathrm{E}$, Hamer $\mathrm{M}$, Tilling $\mathrm{K}$, et al. Sedentary time in relation to cardio-metabolic risk factors: differential associations for self-report vs accelerometry in working age adults. Int $J$ Epidemiol 2012;41:1328-37.
2. Healy GN, Matthews CE, Dunstan DW, et al. Sedentary time and cardio-metabolic biomarkers in US adults: NHANES 2003-06. Eur Heart J 2011;32:590-7.

3. Teychenne M, Ball K, Salmon J. Sedentary behavior and depression among adults: a review. Int J Behav Med 2010;17:246-54.

4. Hamer M, Stamatakis E, Mishra GD. Television- and screen based activity and mental well-being in adults. Am J Prev Med 2010;38:375-80.

5. Atkin AJ, Adams E, Bull FC, et al. Non-occupational sitting and mental well-being in employed adults. Ann Behav Med 2012;43:181-8.

6. Teychenne M, Ball K, Salmon J. Physical activity, sedentary behavior and depression among disadvantaged women. Health Educ Res 2010;25:632-44.

7. Sanchez-Villegas A, Ara I, Guillén-Grima F, et al. Physical activity, sedentary index, and mental disorders in the SUN cohort study. Med Sci Sports Exerc 2010;40:827-34.

8. Lucas M, Mekary R, Pan A, et al. Relation between clinical depression risk and physical activity and time spent watching television in older women: a 10-year prospective follow-up study. Am J Epidemiol 2011;174:1017-27.

9. Hamer M, Stamatakis E. Prospective study of sedentary behavior, risk of depression and cognitive impairment. Med Sci Sports Exerc 2014;46:718-23.

10. Mekary RA, Lucas M, Pan A, et al. Isotemporal substitution analysis for physical activity, television watching, and risk of depression. $A m$ J Epidemiol 2013;178:474-83.

11. van Uffelen JG, van Gellecum YR, Burton NW, et al. Sitting-time, physical activity, and depressive symptoms in mid-aged women. Am J Prev Med 2013;45:276-81.

12. Sloan RA, Sawada SS, Girdano D, et al. Associations of sedentary behavior and physical activity with psychological distress: a cross-sectional study from Singapore. BMC Public Health 2013;13:885.

13. Vallance JK, Winkler EA, Gardiner PA, et al. Associations of objectively-assessed physical activity and sedentary time with depression: NHANES (2005-2006). Prev Med 2011;53:284-8.

14. Joint Health Surveys Unit. The Health Survey for England 2008 Volume 2: methods and documentation. Leeds: The Information Centre for Health and Social Care, 2009. http://www.ic.nhs.uk/pubs/ hse08physicalactivity (accessed May 2010).

15. Stamatakis E, Davis M, Stathi A, et al. Associations between multiple indicators of objectively-measured and self-reported sedentary behaviour and cardiometabolic risk in older adults. Prev Med 2012;54:82-7.

16. Goldberg D, Gater R, Sartorius N, et al. The validity of two versions of the GHQ in the WHO study of mental illness in general health care. Psychol Med 1997;27:191-7.

17. Russ TC, Stamatakis E, Hamer M, et al. Association between psychological distress and mortality: individual participant pooled analysis of 10 prospective cohort studies. BMJ 2012;345:e4933.

18. Aalto AM, Elovainio M, Kivimäki M, et al. The Beck Depression Inventory and General Health Questionnaire as measures of depression in the general population: a validation study using the Composite International Diagnostic Interview as the gold standard Psychiatry Res 2012;197:163-71.

19. Stamatakis E, Hamer M, Mishra GD. Early adulthood television viewing and cardiometabolic risk profiles in early middle age: results from a population, prospective cohort study. Diabetologia 2012;55:311-20.

20. Stamatakis E, Hamer M, Dunstan DW. Screen-based entertainment time, all-cause mortality, and cardiovascular events: population-based study with ongoing mortality and hospital events follow-up. J Am Coll Cardiol 2010;57:292-9.

21. Dowlati $\mathrm{Y}$, Herrmann N, Swardfager W, et al. A meta-analysis of cytokines in major depression. Biol Psychiatry 2010;67:446-57.

22. Hamer M, Poole L, Messerli-Bürgy N. Television viewing, C-reactive protein, and depressive symptoms in older adults. Brain Behav Immun 2013;33:29-32.

23. Rock PL, Roiser JP, Riedel WJ, et al. Cognitive impairment in depression: a systematic review and meta-analysis. Psychol Med 2013;29:1-12.

24. Yoshiuchi K, Inada S, Nakahara R, et al. Stressful life events and habitual physical activity in older adults: 1-year accelerometer data from the Nakanojo Study. Ment Health Phys Activ 2010;3:23-25.

25. Yoshiuchi K, Nakahara R, Kumano $\mathrm{H}$, et al. Yearlong physical activity and depressive symptoms in older Japanese adults: cross-sectional data from the Nakanojo study. Am J Geriatr Psychiatry 2006;14:621-4.

26. Poole L, Steptoe A, Wawrzyniak AJ, et al. Associations of objectively measured physical activity with daily mood ratings and psychophysiological stress responses in women. Psychophysiology 2011;48:1165-72. 
27. Kawada $\mathrm{T}$, Katsumata M, Suzuki $\mathrm{H}$, et al. Actigraphic predictors of the depressive state in students with no psychiatric disorders. $J$ Affect Dis 2007;98:117-20.

28. Janney CA, Richardson CR, Holleman RG, et al. Gender, mental health service use and objectively measured physical activity: data from the National Health and Nutrition Examination Survey (NHANES 2003-2004). Ment Health Phys Activ 2008;1:9-16.

29. Buman MP, Hekler EB, Haskell WL, et al. Objective light-intensity physical activity associations with rated health in older adults. Am J Epidemiol 2010;172:1155-65.

30. Rethorst CD, Wipfli BM, Landers DM. The antidepressive effects of exercise: a meta-analysis of randomized trials. Sports $\mathrm{Med}$ 2009;39:491-511.
31. Moses J, Steptoe A, Mathews A, et al. The effects of exercise training on mental well-being in the normal population: a controlled trial. J Psychosom Res 1989;33:47-61.

32. Puetz TW, Flowers SS, O'Connor PJ. A randomized controlled trial of the effect of aerobic exercise training on feelings of energy and fatigue in sedentary young adults with persistent fatigue. Psychother Psychosom 2008;77:167-74.

33. Puetz TW, O'Connor PJ, Dishman RK. Effects of chronic exercise on feelings of energy and fatigue: a quantitative synthesis. Psycho Bull 2006;132:866-76.

34. Joint Health Surveys Unit. Health Survey for England physical activity validation study: substantive report. Leeds: Information Centre for Health and Social Care, 2007. 